DIW BERLIN

Discussion Papers

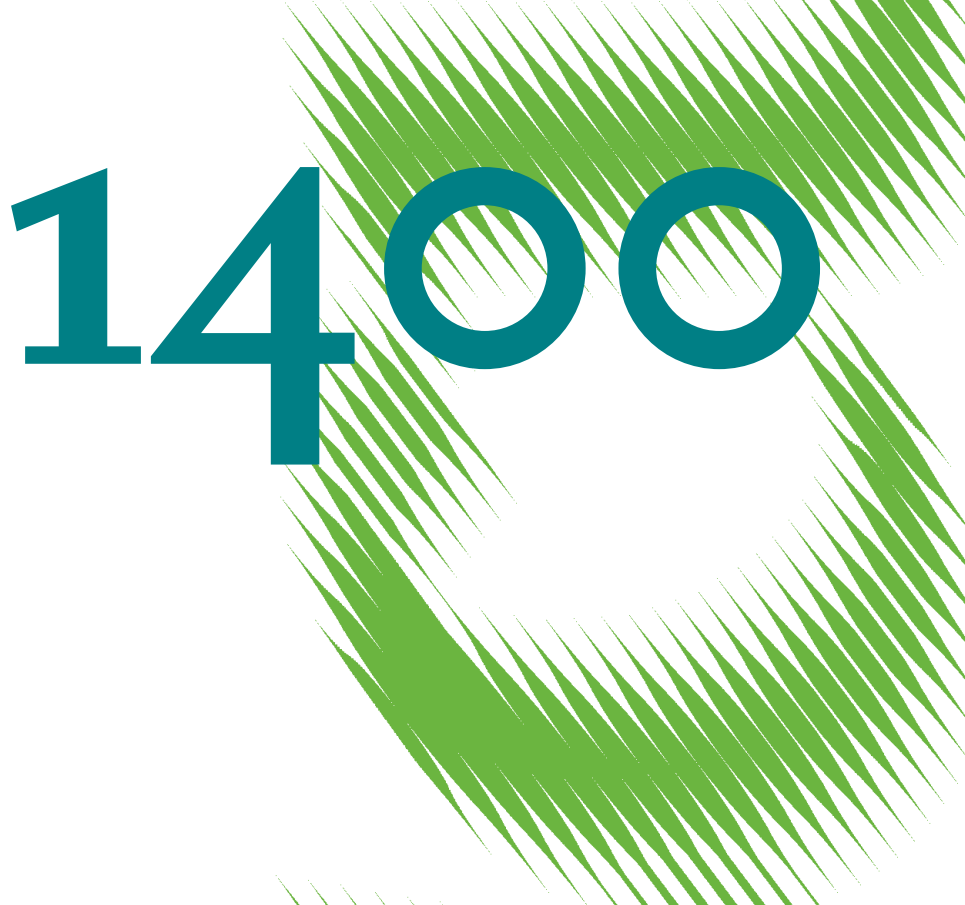

New Cross-Border Electricity Balancing Arrangements in Europe 
Opinions expressed in this paper are those of the author(s) and do not necessarily reflect views of the institute.

IMPRESSUM

(C) DIW Berlin, 2014

DIW Berlin

German Institute for Economic Research

Mohrenstr. 58

10117 Berlin

Tel. +49 (30) $89789-0$

Fax +49 (30) $89789-200$

http://www.diw.de

ISSN electronic edition 1619-4535

Papers can be downloaded free of charge from the DIW Berlin website:

http://www.diw.de/discussionpapers

Discussion Papers of DIW Berlin are indexed in RePEc and SSRN:

http://ideas.repec.org/s/diw/diwwpp.html

http://www.ssrn.com/link/DIW-Berlin-German-Inst-Econ-Res.html 


\title{
New Cross-Border Electricity Balancing Arrangements in Europe*
}

\author{
Casimir Lorenz $z^{\dagger \ddagger}$ and Clemens Gerbaulet ${ }^{\dagger \ddagger}$
}

July 2014

\begin{abstract}
The European electricity system is undergoing significant changes, not only with respect to developments in generation and networks but also the arrangements for the operation of the system. These are specified in the Network Codes endorsed by regulators, network operators and the European Commission with the objective to create an "Internal Energy Market". In 2013, European network operators formulated the Network Code on Electricity Balancing (NC EB) which foresees arrangements to foster cross-border exchange of balancing services with the objective to lower overall costs and to increase social welfare. Assuming that Switzerland adopts the "Electricity Agreement" which would make EU Electricity rulings binding also in Switzerland, we perform an quantitative analysis of the region consisting of Switzerland, Austria, and Germany. To conduct our analysis, we use an electricity market model with a detailed representation of power plants, scheduled power withdrawals and localized imbalances leading to the need to reserve balancing capacity and activate balancing energy. We consider different levels of integration, as outlined in the NC EB. Our results show that coordinated procurement and activation of balancing services lead to cost decreases, but at the same time distributional effects, which might need to be compensated are incurred.
\end{abstract}

JEL Codes: C61, L94, Q40

Keywords: balancing energy markets, regional cooperation, network code electricity balancing

\footnotetext{
*Financial support by the Mercator foundation in the project MASMIE is gratefully acknowledged.

${ }^{\dagger}$ German Institute for Economic Research (DIW Berlin), Department of Energy, Transportation, Environment, Mohrenstraße 58, 10117 Berlin.

${ }^{\ddagger}$ Berlin University of Technology, Workgroup for Infrastructure Policy (WIP), Straße des 17. Juni 135, 10623 Berlin.
} 


\section{Introduction}

One of the European Commission's goals is to establish an internal energy market for Europe. This includes a reformation of the electricity market, laid out to a large extent in Directive 2009/72/EC and Regulation EC No. 714/2009. This makes the European electricity system subject to significant changes, not only with respect to developments in generation and networks but also the arrangements for the operation of the system. These arrangements are defined in Network Codes endorsed by regulators, network operators and the European Commission. In 2013, European network operators formulated the Network Code on Electricity Balancing (NC EB) which foresees arrangements to foster cross-border exchange of balancing services with the objective of lowering overall costs and increasing social welfare. (ENTSO-E, 2013b)

This is of utmost importance since in the long term a high share of renewables will be reached. This could lead to higher balancing needs and lower balancing supply if the current balancing markets design remains unchanged. Borggrefe \& Neuhoff (2011) see the upcoming importance of balancing markets with rising shares of wind penetration and propose a joint provision and adjustment of balancing services. While balancing markets have a much lower volume than the spot market, changes on balancing markets can also influence the spot market price. Wieschhaus \& Weigt (2008) analyze these influences and show that an increasingly competitive balancing market also leads to lower prices on the spot market.

Balancing costs in Germany are relatively constant, although the renewable share is rising (Hirth \& Ziegenhagen, 2013). This can partly be explained by the reformation of the market design in Germany. Surprisingly, this effect was not visible for negative secondary control reserve, where costs went up significantly. Nevertheless dena (2014) and Holttinen et al. (2011) project rising balancing reserve requirements and specific costs for higher renewables shares if the market circumstances do not change.

Balancing power products stabilize the system's frequency of $50 \mathrm{Hertz}$ in the European electricity grid. In general, deviations from the nominal frequency can occur due to unexpected fluctuations in demand or generation. Distinguished by their response time and length of activation, three products are auctioned: primary control (PC), secondary control (SC), and tertiary control (TC).

Currently, these products are auctioned on mainly national markets with partly different procurement mechanisms. In Germany a joint balancing control area with joint coordinated procurement of secondary reserve capacity including all four German transmission system operators (TSOs) was established in 2010. This cooperation was extended in 2012 to the "IGCC - International Grid Control Cooperation", which is limited to the avoidance of counter activation between two countries. Hence there is no joint procurement or activation of SC or TC that could require the alteration of national framework conditions. Additional participants since 2012 are Energinet.dk (Denmark), Swissgrid (Switzerland), ČEPS (Czech Republic), Elia (Belgium), and TenneT TSO B.V. (the Netherlands). In 2014 the cooperation was expanded by APG (Austria) and there are future plans for ongoing growth (IGCC, 2014). Further regional cooperation on 
the tertiary balancing energy markets of Germany and Austria already exists, as some Austrian power plants can bid into the German market.

In the literature several studies have treated the issue of cross-border balancing cooperation. Van der Veen et al. (2010) give an overview on main cross-border balancing agreements and perform a qualitative analysis on seven arrangements. They conclude that these are generally positive but there are uncertainties regarding their impact depending on the resulting detailed balancing market design.

A study for the European Commission analyzes the impacts of a European balancing market. It studies different approaches to handle cross-border exchanges of balancing services by applying empirical methods as well as quantitative simulations. The results prove a gain in social welfare and additional advantages for the integration of Variable Renewable Energies (VREs). To reach this goal the study recommends a TSO-to-TSO platform with a Common Merit Order List (CMOL), harmonization of key elements and "appropriate" bidding blocks. However the applied methodologies differ widely from the one applied in this paper and are mostly focused on historical bid data. (DG ENER, 2013)

Gebrekiros et al. (2013) develop a model where bidding prices for balancing capacity are generated based on the power plants' opportunity cost resulting from the day-ahead market, which is influenced by intermittent renewable infeed, to evaluate the benefits of cross-border reserve procurement. However, in a test case only minor cost reductions are observed.

Integration of the balancing markets in northern Europe has been dealt with in the literature in several studies. Abbasy et al. (2009) analyze the effect of integration balancing markets of Northern Europe. They show that balancing cost can be decreased by $€ 100$ million in the region by increased integration. While overall cost are reduced, balancing power prices remained stable on average. A similar question is analyzed by Jaehnert \& Doorman (2010). The authors show that increased integration of the Nordic and German balancing markets shows positive effects, but these are dependent on assumptions regarding the cost of regulation services. Farahmand \& Doorman (2012) estimate cost savings of up to $€ 400$ million per year resulting from an integration of the Nordic balancing market with the German balancing market. The positive effects of cross-border cooperation in providing balancing services is furthermore shown by an agent-based analysis for different agreements for integrating the Dutch, German and Nordic balancing markets by Van der Veen et al. (2011). Results indicate a promising $50 \%$ reduction of balancing cost resulting from the implementation of a common merit order list. Furthermore, Abbasy et al. (2011) analyze the effects of of BSP ${ }^{1}$-TSO trading (i.e. foreign bidding) between Norway and the Netherlands. To simulate the change in market prices an agent-based model, developed in MATLAB, is used. They conclude, that there is no general answer to whether a BSP-TSO model would result in too much shifted capacity (therefore increasing prices in the cheaper market) because this is dependent on the current situation of the spot market. The usage of an agent-based model allows to introduce strategic behavior and different players. However it requires assumptions on

\footnotetext{
${ }^{1}$ Balancing Service Provider
} 
the behavior of players, which can influence results to a great extent. Farahmand et al. (2012) compare the effects of a non-integrated and a fully-integrated balancing market in the Nordic region for a 2030 scenario. They apply a two stage approach to model the spot and balancing market, which is similar to the approach applied in this paper. Results show that there are possible cost saving opportunities due to balancing market integration that allow for less activation and cheaper reservation of balancing capacity.

Regional cooperation in the procurement of tertiary balancing capacity in the alpine region has been analyzed by Gerbaulet et al. (2012) with the result, that common procurement leads to cost decreases in the region. Bilateral cooperations can also lead to a decrease in total cost. The authors note that optimal allocation of interconnector capacity for the spot market and balancing services might gain significance in the future.

Besides the benefits of cooperation described above, pursuing cross-border balancing agreements might be a challenging task. A comprehensive study by Tractebel (2009) analyzes a pathway towards cross-border balancing agreements in Europe and demonstrates possible obstacles. Main prerequisites of cross-border harmonization are identified as common technical characteristics of balancing services and gate closure times, a common remuneration mechanism for balancing services, and a harmonization of imbalance settlement mechanisms. Possible inefficiencies and distortions due to insufficient harmonization of national market designs are analyzed by Vandezande et al. (2008). They recommend an implementation of cross-border balancing agreements with very low prerequisites to allow for a fast and functioning realization. Intensified harmonization should be done in a later stage.

Building on the prevailing literature we focus on the region of Austria, Germany and Switzerland and analyze possible effects of the proposed NC EB for secondary and tertiary balancing capacity, taking into account the cross-border lines and potential competing allocation objectives of the different energy markets.

The remainder of this paper is structured as follows: section 2 outlines the current political discussion regarding the new NC EB. Section 3 describes the methodology applied in this paper and underlying assumptions. The mathematical formulation of the model is explained in section 4. Section 5 describes the scenarios applied in the model. The data and application are presented in section 6. In Section 7 the quantitative results are discussed. Section 8 draws conclusions.

\section{Current Political Discussion}

In 2012 the Agency for the Cooperation of Energy Regulators (ACER) published the Framework Guidelines on Electricity Balancing (ACER, 2012). Based on theses guidelines the ENTSO-E was invited by the European Commission to draft a Network Code (ENTSO-E, 2013b). As of summer 2014 the ENTSO-E's latest draft of the NC EB is the December 2013 edition, which ACER commented on in March 2014 (ACER, 2014).

In the following paragraphs the ENTSO-E's Network Code on Electricity Balancing Draft (ENTSO-E, 2013b) is presented. As stated above, one main target of the new NC EB is to promote the exchange of balancing services. To foster this, every TSO 
should form a so called "Coordinated Balancing Area" (CoBA) with at least one TSO operating in a different member state. $\mathrm{A} \mathrm{CoBa}$ is a cooperation between two or more TSOs with the aim of the exchange of balancing services. Furthermore, this implies the exchange of at least one standardized product or the application of imbalance netting. The created CoBA should be open to any other TSO.

The NC EB also proposes models for the exchange of balancing energy for frequency restoration reserves, replacement reserves and imbalance netting. These are formulated for a regional perspective but also for a European perspective with longer time horizons in mind. Furthermore it specifies the role of TSOs, balance responsible parties, and the prerequisites for standardized products. In addition, different procurement processes are specified in the NC EB.

If a CoBA decides to implement joint procurement of balancing capacity, the selection of the balancing capacity bids shall be performed by a so called "Capacity Procurement Optimization Function". This function is an algorithm that seeks to minimize the overall procurement costs for all commonly procuring TSOs within a CoBA. However, for joint procurement of balancing capacity a CoBA is not necessarily needed.

For the procurement of balancing energy all TSOs must harmonize the pricing method for at least one standardized balancing energy product. It should be based on marginal pricing. This is in contrast to the current situation in Austria, Germany and Switzerland where pay-as-bid pricing is used for capacity bids in SC and TC markets. However for non-standardized products or those with a positive cost-benefit-analysis pay-as-bid can still be used.

The activation of balancing energy should be coordinated by a so called "Activation Optimization Function". Similar to the "Capacity Procurement Optimization Function" this algorithm optimizes the activation of balancing energy bids from a CMOL, which takes into account operational and transmission restrictions.

In contrast to the current legislation, TSOs should have the right to reserve crossborder-capacity for balancing when it would be socio-economically beneficial. Furthermore important aspects (gate closure times, energy only bids) that allow for the integration of VREs are addressed in the NC EB but are not relevant for our analysis. (ENTSO-E, 2013b)

The comments on the NC EB from March 2014 by ACER are partly critical as the NC EB is not in line with the Framework Guidelines (ACER, 2012) previously provided by ACER. The NC EB apparently does not respect the timelines proposed by ACER and puts to much weight on voluntary approaches, while ACER demands clear and legally binding requirements for the TSOs. Furthermore the NC EB is not ambitious enough when it comes to harmonizing of core elements. Hence, according to ACER, the NC EB misses opportunities to decrease the need for TSOs to balance the system and to enhance competition in the balancing markets. (ACER, 2014) 
Table 1: Model steps

\begin{tabular}{ll}
\hline Step & Description \\
\hline 1. Spot market & $\begin{array}{l}\text { The spot market dispatch is calculated without balancing re- } \\
\text { strictions to determine the cross-border flows. }\end{array}$ \\
2. Reservation & $\begin{array}{l}\text { Spot market dispatch is recalculated given balancing capacity } \\
\text { requirements and the predetermined cross-border flows. Capacity } \\
\text { for cross-border activation is reserved depending on the scenario. } \\
\text { Balancing energy is called given the reservation done in the } \\
\text { previous step. This is either conducted for each region or the } \\
\text { whole balancing area depending on the scenario. }\end{array}$ \\
\hline
\end{tabular}

\section{Methodology}

In this paper we analyze the benefits stemming from regional cooperation between Austria, Germany, and Switzerland in the procurement of balancing services taking into account the suggestions of the NC EB. We analyze the effects on secondary and tertiary control and neglect primary control.

We apply a model that determines the cost-minimal power plant dispatch in the spot market under the assumption of perfect competition. The reservation of balancing capacity is conducted taking into account the overall structure and timing of the balancing energy calls to minimize the system cost.

In our model there are two factors inducing costs when reserving balancing capacity: On the one hand opportunity costs occur due to balancing restrictions on the available generation capacity as capacity is either reserved in a power plant in case of positive capacity reservation, or a must-run condition is introduced in case of negative capacity reservation. On the other hand calling balancing energy leads to costs, because additional fuel is required or deviations from the optimal power plant dispatch occur. Pumped storage and hydro reservoirs can also participate in the balancing energy market. Although no actual fuel cost occur in these plants, the connected nature of the electricity system leads to opportunity costs that are taken into account as well.

This paper neglects price markups for balancing capacity as it focuses on the inefficiencies that exist in the balancing markets devoid of strategic behavior. Historical price markups, that are used in the majority of the existing literature, might distort the model results significantly in case of market integration, as the markups are usually not endogenous to the model. This could lead to an overestimation of the cost saving potential. Therefore our results will show reduced benefits, as the model setting is different in comparison to the existing literature.

We formulate a Mixed Integer Program (MIP) as a multi-step model. The steps involved are shown in table1. For all steps the same model is used, but relevant variables and parameters are fixed or set to zero based on each step's goal. In step 1 the spot market is solved without balancing energy reservation restrictions. This determines the 


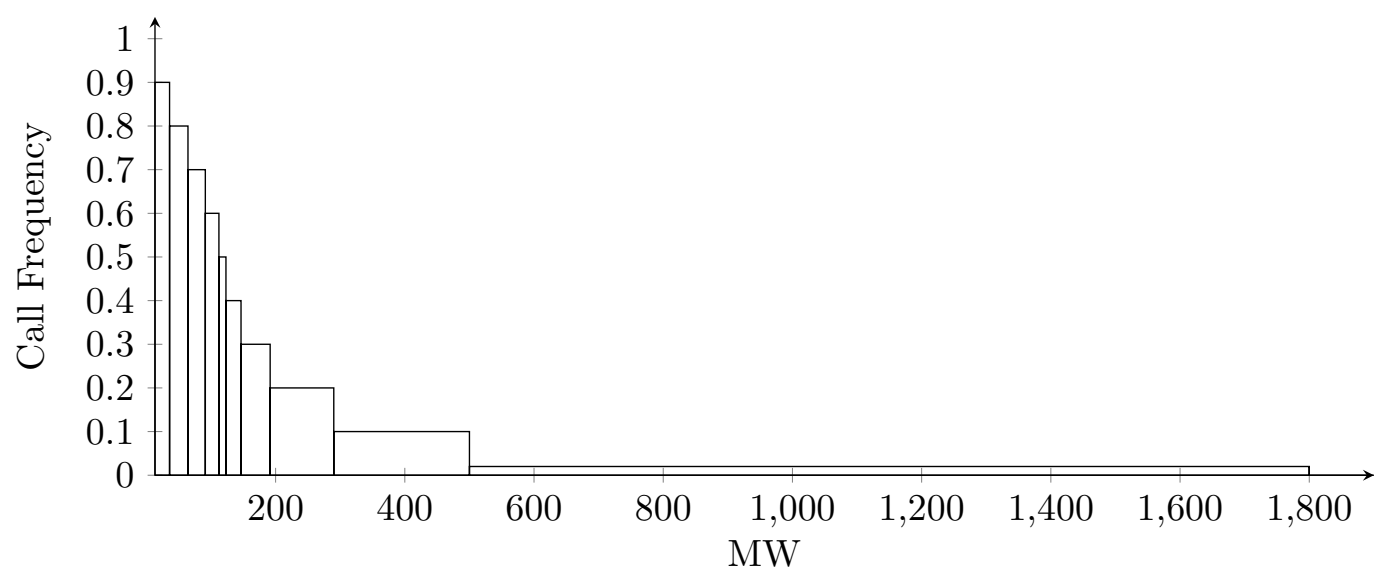

Figure 1: Exemplary call frequency and block size for positive SC

theoretical spot market flows between the model regions, which are fixed in the subsequent steps. This step is necessary because in the next steps the flows of balancing energy on the borders are taken into account as well. Leaving the spot market flows unfixed would enable the model to counter-reserve balancing capacity that would negatively impact the spot market flows. The next step 2 optimizes the spot market and reservation of the balancing capacities taking into account the predetermined spot market flows and anticipated balancing calls. Finally in step 3, the dispatch including the call of balancing energy is optimized. Here, the variables determining the reservation of balancing capacity are fixed in the model. Only power plants with reserved capacity can be dispatched for balancing energy calls by the model. No uncertainty about future spot market outcomes is integrated at this point, hence load and RES feed-in are certain for all hours of the model.

To resemble the uncertainty of how much balancing power is actually called (i.e. activated), we separate the balancing reserves into blocks differentiated by their frequency of being called based on historical time series. This methodology approximates the actual call distribution, as a small amount of balancing power is almost always realized, but the maximum reserved capacity is called only in a few hours per year. The model uses this information to determine which power plants should be used for the reservation of balancing energy. Hence the anticipated cost for the call is dependent on the call frequency and has an impact on the total system costs. Exemplary frequencies for positive $\mathrm{SC}$ are shown in figure 1. We derive the distribution of these blocks using historical time series data that is also part of the model input described in section 6. The sum of each block's size in MW multiplied by the frequency equals the average call values in MW. This ensures that the model's anticipation of the average balancing energy calls is correct.

In the current market design balancing capacity is reserved regularly for time periods between four hours and one week, depending on the product and region. Furthermore this reservation is allocated to the bidding firms. The firms can optimize the dispatch of their power plant portfolio at the time of delivery of the balancing energy. In our 
model we abstract from this setting, thus balancing capacity can be reserved for each power plant and hour separately. This results in a situation similar to a single big firm participating in a cost minimizing behavior on the balancing markets.

\section{Model Implementation}

The model's objective is to minimize total system costs, while clearing the spot market as well as the balancing market for the two balancing power products SC and TC. The model is solved in GAMS (General Algebraic Modeling System) using the commercial solver CPLEX.

The mathematical formulation can be found in equations (1) to (51). The objective is to minimize the sum of generation, startup, shutdown and balancing cost (1- 6).

$$
\min C o s t=\operatorname{Cost}^{\text {gen }}+\text { Cost }^{\text {start }}+\text { Cost }^{\text {down }}+\text { Cost }^{\text {resv }}+\text { Cost }^{\text {call }}
$$

s.t.

$$
\begin{aligned}
\text { Cost }^{\text {gen }} & =\sum_{c, t} m c_{c} \cdot G_{c, t}^{\text {Spot }} \\
\text { Cost }^{\text {start }} & =\sum_{c, t} U P_{c, t} \cdot c_{c}^{\text {start }}+\sum_{u, t} F r q_{u, t}^{\text {max }} \cdot c_{u}^{\text {start }} \\
\text { Cost }^{\text {down }} & =\sum_{c, t} D N_{c, t} \cdot c_{c}^{\text {down }}+\sum_{u, t} F r q_{u, t}^{\text {max }} \cdot c_{u}^{\text {down }} \\
\text { Cost }^{\text {resv }} & =\sum_{b, b l, c, t} m c_{c} \cdot\left(G_{c, t, b l, b}^{\text {resv,pos }}-G_{c, t, b l, b}^{\text {resv,neg }}\right) \cdot f r q_{b l, b} \\
\text { Cost }^{\text {call }} & =\sum_{b, c, t} m c_{c} \cdot\left(G_{b, c, t}^{\text {call,pos }}-G_{b, c, t}^{\text {call,neg }}\right)
\end{aligned}
$$

The total system costs (1) include variable costs of generation (2), start-up and shutdown costs (3), (4) and the costs for providing balancing power (5) and (6). The variable cost of generation is defined as the generation $G_{c, t}^{s p o t}$ of all conventional power plants $c$ and time steps $t$ multiplied by the plants' marginal production cost $m c_{c}$. Start-up cost $c_{c}^{\text {start }}$ occur when a plant assumes production and was in a shut-down state in the previous time step. Then, the binary variable $U P_{c, t}$ has the value 1 . During the reservation phase the expected start up probability of fast-starting power plants $u$, which is a subset of all conventional power plants $c$, is also taken into account. Shut-down cost occur analogously. For fast-starting power plants, that are not participating in the spot market at the time of call, we assume that these plants provide balancing power for a short time period and shut down afterward. Therefore the shut-down cost $c_{u}^{\text {down }}$ are taken into account as well during the reservation phase. The reservation of positive or negative balancing capacity $G_{c, t, b l, b}^{r e s v, p o s}$ and $G_{c, t, b l, b}^{r e s v e g}$ for the balancing power products $b$ and blocks $b l$ incurs opportunity cost $m c_{c}$ multiplied with the block's specific call frequency $f r q_{b l, b}$ in the model, as the capacity reservation reduces the available capacity in the spot markets. 
The balancing energy calls $G_{b, c, t}^{\text {call,pos }}$ and $G_{b, c, t}^{\text {call,neg }}$ are accounted for by the power plants' marginal cost $m c_{c}$.

\section{Market Clearing}

$$
\begin{aligned}
& 0=q_{r, t}^{s p o t}-\sum_{c \in r} G_{c, t}^{s p o t}+\sum_{s}\left(P S P_{s, t}^{D}-P S P_{s, t}^{G}\right) \\
& -g_{r, t}^{\text {wind }}-g_{r, t}^{\text {sol }}-g_{r, t}^{\text {bio }}+\sum_{r r} F_{r, r r, t}^{s p o t} \\
& q_{b, b l, r, t}^{\text {resv }, p o s}=\sum_{p \in r} G_{p, t, b l, b}^{r e s v, p o s}-\sum_{r r} F_{b, b l, r, r r, t}^{r e s v, p o s} \quad \forall t, r, b l, b \\
& q_{b, b l, r, t}^{r e s v, n e g}=\sum_{p \in r} G_{p, t, b l, b}^{r e s v, n e g}-\sum_{r r} F_{b, b l, r, r r, t}^{r e s v, n e g} \quad \forall t, r, b l, b \\
& q_{b, r, t}^{\text {call,pos }}=\sum_{p \in r} G_{b, p, t}^{\text {call,pos }}-\sum_{r r} F_{b, r, r r, t}^{\text {call,pos }} \quad \forall b, r, t \\
& q_{b, r, t}^{\text {call,neg }}=\sum_{p \in r} G_{b, p, t}^{\text {call,neg }}-\sum_{r r} F_{b, r, r r, t}^{\text {call,neg }} \quad \forall b, r, t
\end{aligned}
$$

The spot market is cleared by leveling load $q_{r, t}^{s p o t}$, generation $G_{c, t}^{s p o t}$, storage $P S P_{s, t}^{D}, P S P_{s, t}^{G}$, renewable feed-in $g_{r, t}^{\text {wind }}, g_{r, t}^{\text {sol }}, g_{r, t}^{\text {bio }}$ and exchange flows $F_{r, r r, t}^{s p o t}$ for all time steps $t$ and regions $r$, as stated in (7). Markets for positive and negative balancing capacity are cleared separately for each product $b$ and block $b l$, by leveling demand $q_{b, b l, r, t}^{r e s v, p o s}, q_{b, b l, r, t}^{r e s v}$, reserves $G_{p, t, b l, b}^{r e s v, p o s}, G_{p, t, b l, b}^{r e s v, n e g}$, and cross-border flows to or from other regions $F_{b, b l, r, r r, t}^{b a l, p o s}, F_{b, b l, r, r r, t}^{b a l, n e g}$ This is shown in (8) and (9) for the reservation and (10) and (11) for the call of balancing energy.

\section{Generation restrictions}

$$
\begin{aligned}
G_{c, t}^{s p o t} & \leq g_{c}^{\text {max }}-\sum_{b l, b} G_{c, t, b l, b}^{r e s v, p o s} & & \forall c, t \\
G_{c, t}^{s p o t} & \geq g_{c}^{\text {min }} \cdot O N_{c, t}+\sum_{b l, b} G_{c, t, b l, b}^{r e s v, n e g} & & \forall c, t \\
G_{o, t}^{s p o t} & \leq O N_{o, t} \cdot g_{o}^{\text {max }}-\sum_{b l, b} G_{o, t, b l, b}^{r e s v, p o s} & & \forall o, t \\
G_{u, t}^{s p o t} & \leq O N_{u, t} \cdot g_{u}^{\max } & & \forall u, t \\
D N_{c, t}+O N_{c, t} & =U P_{c, t}+O N_{c, t-1} & & \forall c, t
\end{aligned}
$$

A power plant's generation $G_{c, t}^{s p o t}$ and balancing reservation $G_{c, t, b l, b}^{r e s v, p o s}, G_{c, t, b l, b}^{r e s v e g}$ is constrained by its minimal and maximal generation capacity (12), (13). Slow starting power plants $o$ have to be online to provide balancing power (14) while fast starting power plants $u$ must only be online when providing energy for the spot market (15). In case 
of activation of reserve energy we assume that these power plants can reach the desired output levels within time from a shutdown state. Equation (16) tracks the plant's status for start-up and shut-down costs and enforces the plant to start up when providing balancing power.

\section{Ramping}

$$
\begin{array}{ll}
0 \leq r_{c}^{u p} \cdot g_{c}^{\text {max }}-G_{c, t}^{s p o t}+G_{c, t-1}^{s p o t}+\sum_{b l, b} G_{c, t-1, b l, b}^{r e s v, n e g}-\sum_{b l, b} G_{c, t, b l, b}^{r e s v, p o s} & \forall c, t \\
0 \leq r_{c}^{\text {down }} \cdot g_{c}^{\text {max }}-G_{c, t-1}^{\text {Spot }}+G_{c, t}^{\text {spot }}+\sum_{b l, b} G_{c, t, b l, b}^{r e s v, n e g}-\sum_{b l, b} G_{c, t-1, b l, b}^{r e s v, p o s} & \forall c, t
\end{array}
$$

The power plants' ramping restrictions are included in (17) and (18). These equations limit the change of a power plant's production levels between time steps. For ramping, only the limiting balancing reservations are included, as otherwise the model would be able to weaken the ramping restrictions by reserving balancing capacity in the reverse direction.

\section{Reserve restrictions}

$$
\begin{aligned}
G_{c, t}^{\text {spot }} & \geq \sum_{b, b l} G_{b, b l, c, t}^{r e s v, n e g} & & \forall c, t \\
G_{b, b l, c, t}^{r e s v, p o s} & \leq\left(S B_{b, b l, u, t}+O N_{c, t}\right) \cdot g_{u}^{\max } & & \forall b, b l, c, t \\
F r q_{u, t}^{\max } & \geq S B_{b, b l, u, t} \cdot f r q_{b, b l} & & \forall b l, u, t, b \\
1 & \geq S B_{b, b l, u, t}+O N_{u, t} & & \forall b l, u, t, b
\end{aligned}
$$

Equations (19) to 22 describe the restrictions that determine how much of a plant's capacity can be reserved for balancing. The amount of negative reserved balancing power must always be smaller that the spot market generation of the power plant (19). This enforces power plants to be online and to participate in the spot market in order to provide negative balancing power. Slow starting power plants $o$ must be online to provide positive balancing power as well, whereas fast starting power plants $u$ can be in standby mode 20. Fast starting plants that are not generating but providing balancing power will incur their start-up and shut-down costs according to their expected call frequency (21). Equation 22 ensures that plants can only either be online or in standby mode.

\section{Call restrictions}

$$
\begin{aligned}
G_{b, p, t}^{\text {call,pos }} & \leq \sum_{b l} G_{b, b l, p, t}^{\text {resv,pos }} & \forall b, p, t \\
G_{b, p, t}^{\text {call,neg }} & \leq \sum_{b l} G_{b, b l, p, t}^{r e s v, n e g} & \forall b, p, t
\end{aligned}
$$




$$
\sum_{b} G_{b, u, t}^{\text {call,pos }} \leq g_{u}^{\max } \cdot O N_{u, t} \quad \forall u, t
$$

When reserve energy is called, the positive and negative call must always be smaller than the reserved amount for each power plant, hour and product as shown in (23) and (24). Equation 25) ensures that fast starting plants must start up to provide balancing energy. Note that the status of the power plants is not transferred between the stages of the multi-stage model but redetermined each stage. A fast-starting power plant that is in "Standby" in the reservation stage with reserved capacity might be set to "Online" during the call stage. This way the actual startup cost of fast starting power plants can be accounted for in the model when the calls take place.

\section{Storage restrictions}

$$
\begin{aligned}
& P S P_{s, t}^{L}=P S P_{s, t-1}^{L}+P S P_{s, t}^{D} \cdot \eta_{s}-P S P_{s, t}^{G}+g_{s, t}^{\text {nat }}-P S P_{s, t}^{\text {discard }} \\
& +\sum_{b l, b}\left(G_{s, t, b l, b}^{r e s v, n e g, A}-G_{s, t, b l, b}^{r e s v, p o s, P}\right) \cdot f r q_{b l, b} \cdot \eta_{s} \\
& -\sum_{b l, b}\left(G_{s, t, b l, b}^{r e s v, p o s, A}-G_{s, t, b l, b}^{r e s v, n e g, P}\right) \cdot f r q_{b l, b} \\
& -\sum_{b} G_{b, s, t}^{\text {call,pos }}+\sum_{b} G_{b, s, t}^{\text {Call,Neg }} \cdot \eta_{s} \\
& G_{s, t, b l, b}^{r e s v, p o s}=G_{s, t, b l, b}^{r e s v, p o s, A}+G_{s, t, b l, b}^{r e s v, p o s, P} \\
& G_{s, t, b l, b}^{r e s v, n e g}=G_{s, t, b l, b}^{r e s v, \text { neg }, A}+G_{s, t, b l, b}^{r e s s, \text { neg }, P} \\
& v_{s}^{\max } \geq P S P_{s, t}^{G}+\sum_{b l, b} G_{s, t, b l, b}^{r e s v, p o s, A} \\
& w_{s}^{\max } \geq P S P_{s, t}^{D}+\sum_{b l, b} G_{s, t, b l, b}^{r e s v, n e g, A} \cdot \eta_{s} \\
& 0 \geq l_{s}^{\text {min }}-P S P_{s, t-1}^{L}-\left(P S P_{s, t}^{D}-\sum_{b l, b} G_{s, t, b l, b}^{r e s v, p o s, P}\right) \cdot \eta_{s} \\
& +P S P_{s, t}^{G}+\sum_{b l, b} G_{s, t, b l, b}^{r e s v, p o s, A} \\
& 0 \leq l_{s}^{\max }-P S P_{s, t-1}^{L}-\left(P S P_{s, t}^{D}+\sum_{b l, b} G_{s, t, b l}^{r e s v, n e g, A}\right) \cdot \eta_{s} \\
& +P S P_{s, t}^{G}-\sum_{b l, b} G_{s, t, b l, b}^{r e s v e g, P} \\
& \forall s, t \\
& \forall s, t, b l, b \\
& \forall s, t, b l, b \\
& \forall s, t \\
& \forall s, t \\
& \forall s, t \\
& \forall s, t \\
& P S P_{s, t}^{G} \geq \sum_{b l, b} G_{s, t, b l, b}^{\text {resv,neg,P }} \\
& \forall s, t \\
& P S P_{s, t}^{D} \geq \sum_{b l, b} G_{s, t, b l, b}^{r e s v, p o s, P}
\end{aligned}
$$


In our model pumped hydro storage plants $s$ take part in the balancing market. Equation (26) describes the storage level $P S P_{s, t}^{L}$ for every storage plant $s$ that is dependent on the historic storage level $P S P_{s, t-1}^{L}$, pumping $P S P_{s, t}^{D}$ and generation activities $P S P_{s, t}^{G}$, and forecasted or realized balancing power usage. In (27) and (28) the reserved capacities are split up in active and passive reservations as positive balancing power can be provided either by increasing generation or by reduced pumping while negative balancing power can be provided by less generation or increased pumping. Equations (29) to (34) limit the pumping, generation, and storage level as well as reserved balancing power.

\section{Flow Restrictions}

$$
\begin{aligned}
& f_{r, r r}^{\max } \geq F_{r, r r, t}^{s p o t}+\sum_{b, b l} F_{b, b l, r, r r, t}^{r e s v}-\sum_{b, b l} F_{b, b l, r, r r, t}^{r e s v, \text { neg,le0 }} \quad \forall r, r r, t \\
& -f_{r, r r}^{\max } \leq F_{r, r r, t}^{\text {Spot }}+\sum_{b, b l} F_{b, b l, r, r r, t}^{r e s v, p o s, l e 0}-\sum_{b, b l} F_{b, b l, r, r r, t}^{\text {resv,neg }, g e 0} \quad \forall r, r r, t \\
& F_{r, r r, t}^{s p o t}=-F_{r r, r, t}^{S p o t} \quad \forall r, r r, t \\
& F_{b, b l, r, r r, t}^{r e s v, p o s}=-F_{b, b l, r r, r, t}^{r e s v, p o s} \\
& \forall b, b l, r, r r, t \\
& F_{b, b l, r, r r, t}^{r e s v, \text { neg }}=-F_{b, b l, r r, r, t}^{r e s v, n e g} \\
& F_{b, r, r r, t}^{\text {call,pos }}=-F_{b, r r, r, t}^{\text {call,pos }} \\
& F_{b, r, r r, t}^{\text {call }, \text { neg }}=-F_{b, r r, r, t}^{\text {call }, \text { eg }} \\
& F_{b, b l, r, r r, t}^{r e s v, g o s} \geq F_{b, b l, r, r r, t}^{r e s v, p o s} \\
& F_{b, b l, r, r r, t}^{r e s, \text { neg }, g e 0} \geq F_{b, b l, r, r r, t}^{r e s v, \text { neg }} \\
& F_{b, b l, r, r r, t}^{r e s v, p o s, l e 0} \leq F_{b, b l, r, r r, t}^{r e s v, p o s} \\
& F_{b, b l, r, r r, t}^{r e s v, \text { neg }, l e 0} \leq F_{b, b l, r, r r, t}^{r e s v, n e g} \\
& \sum_{b l} F_{b, b l, r, r r, t}^{R e s v, \text { Pos, ge } 0} \geq F_{b, r, r r, t}^{\text {call,pos }} \\
& \sum_{b l} F_{b, b l, r, r r, t}^{r e s, p o s, l e 0} \leq F_{b, r, r r, t}^{c a l l, p o s} \\
& \sum_{b l} F_{b, b l, r, r r, t}^{r e s v, \text { neg }, g e 0} \geq F_{b, r, r r, t}^{\text {call,neg }} \\
& \sum_{b l} F_{b, b l, r, r r, t}^{r e s v, n e g, l e 0} \leq F_{b, r, r r, t}^{c a l l, n e g}
\end{aligned}
$$

In our model we distinguish three types of flows: Spot market flows $F_{r, r r, t}^{s p o t}$, flow reservation of balancing capacity $F_{b, b l, r, r r, t}^{r e s v}$, and flows induced by the calls of balancing energy $F_{b, r, r r, t}^{c a l l}$. The maximum flows between regions are limited in the positive (35) and in the negative direction (36). These flows consist of spot market flows as well as reserved capacity for balancing purposes if available in the scenario. Equations 37 to 41 ensure model symmetry. In order to avoid model-induced counteracting for the possible balancing 
flows only the positive or the negative part is included in these equations. Hence counterbalancing-flows can not increase the flow limit. The flows induced by the call of balancing energy must always be lower than the reserved capacity as show in (46) to (49).

\section{Further restrictions}

$$
\begin{aligned}
F_{b, b l, r, r r, t}^{\text {Resv }, \text { ose } 0}, F_{b, b l, r, r r, t}^{\text {Resv }, \text { Neg } 0}, P S P_{s, t}^{D}, P S P_{s, t}^{G}, P S P_{s, t}^{L} & \geq 0 \\
G_{b, b l, c, t}^{\text {Resves }}, G_{b, b l, c, t}^{\text {ResvNeg }}, G_{b, p, t}^{\text {Call,Pos }}, G_{b, p, t}^{\text {Call,Neg }}, F r q_{u, t}^{\text {max }} & \geq 0 \\
F_{b, b l, r, r r, t}^{\text {Resv }, \text { Pos }, l e 0}, F_{b, b l, r, r r, t}^{\text {Resv }, \text { e } 0} & \leq 0
\end{aligned}
$$

Equations (50) and (51) ensure positive or negative values for some variables in the model.

The problem is solved in a rolling planning approach. This enables us to optimize over a long time horizon. Therefore every rolling planning period is optimized consecutively with inter-temporal constraints linking the different rolling planning periods. To generate storage levels and associated limitations for the starting and end period of each rolling planning period, we solve a limited version of the model for the entire model year prior to the actual calculations. This is necessary because large-scale reservoirs not only optimize their dispatch on a day-to-day basis but the reservoir level and inflows into these reservoirs are very different over the course of a year.

\section{Scenarios}

We study different levels of balancing market integration as suggested in the current version of the NC EB: i. No Cooperation, ii. Imbalance Netting only, iii. Joint Activation across borders, and iv. Full Cooperation. A social planner is assumed whose objective is to minimize total system cost while taking into account generation restrictions, reserve restrictions and flow limitations between different countries.

i. In the scenario No Cooperation every country procures and calls balancing services on its own. Cross-border flows on the spot market exist but the balancing markets are separated.

ii. The scenario Imbalance Netting adds limited cooperation between countries during the activation phase of balancing energy. Procurement of balancing capacity takes place nationally like in scenario i., but imbalances are netted between countries when call for balancing energy occur. This avoids unnecessary counteracting between countries.

iii. In the Joint Activation scenario this cooperation is further extended and the activation of balancing energy is coordinated between countries. If cross-border capacity is available, balancing energy can be activated within the country with the lowest cost. The procurement remains separate for each country. 
iv. In the Full Cooperation scenario the coordination extends to the procurement of balancing capacity, building on the setup of scenario Joint Activation. The capacity reservation is conducted for the entire region given cross-border capacity restrictions. We alter the multi-stage model slightly, as steps 1 . and 2. are combined into a single step. Hence the reservation of capacity for cross-border balancing flows competes with the spot market flows. This allows for interesting insights into the value of each kind of cross-border capacity, as the model determines the cost-minimal balance between spot market and balancing flow reservation.

The overall model structure is identical for all scenarios. The scenarios are differentiated by the available transfer capacity for balancing purposes and the netting of imbalances between countries. Spot market flows are only limited by the available Net Transfer Capacities (NTCs) in all stages.

\section{Data and Application to Model Region}

We apply the model to our region of interest consisting of Switzerland, Austria and Germany as shown in figure 2. Liechtenstein is also part of this region and incorporated into Switzerland for our analysis. No surrounding countries are considered and possible exchange flows are not taken into account.

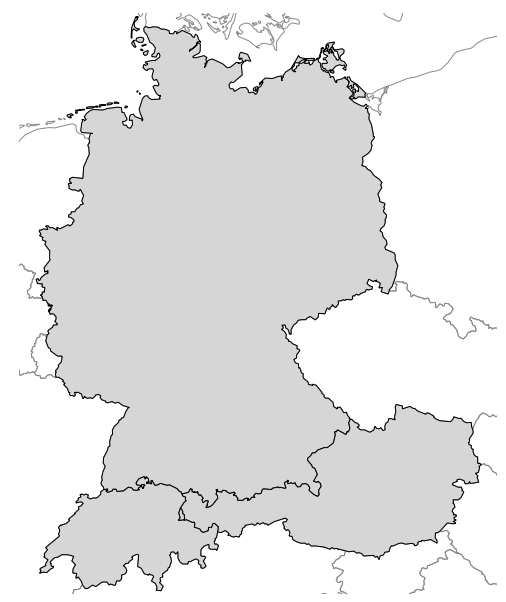

Figure 2: Model Region

Where possible, we use data available to the public. Load, balancing power reserve requirements, and balancing energy calls are based on historical time series from 2013. Four exemplary weeks are selected $\left(2^{\text {nd }}, 16^{\text {th }}, 28^{\text {th }}\right.$, and $41^{\text {st }}$ week $)$ to represent the divergence of renewable feed-in and load over time. Renewable feed-in time series are based on TSO data for Germany from 50Hertz (2013), Amprion (2013), TenneT (2013), and TransnetBW (2013). For Austria and Switzerland the feed-in time series are approximated based on installed capacities and weather data due to the lack of official data. Hydro inflows for Austria are based on E-Control (2013) and for Switzerland on 


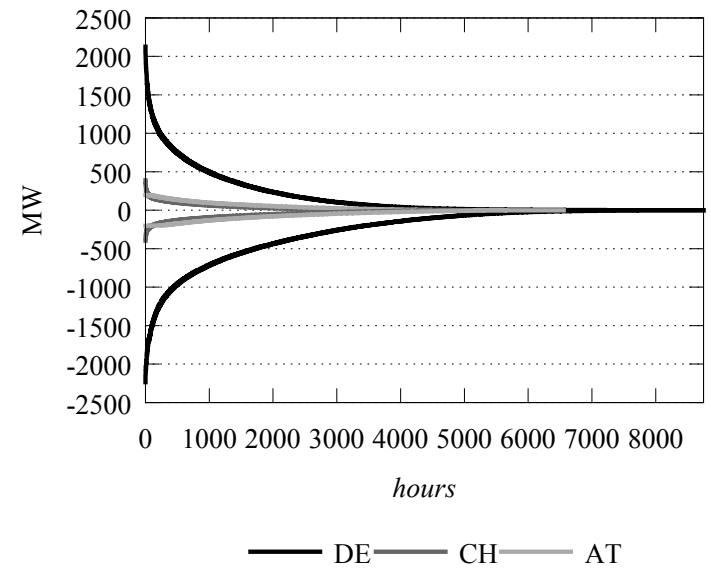

(a) Secondary Capacity

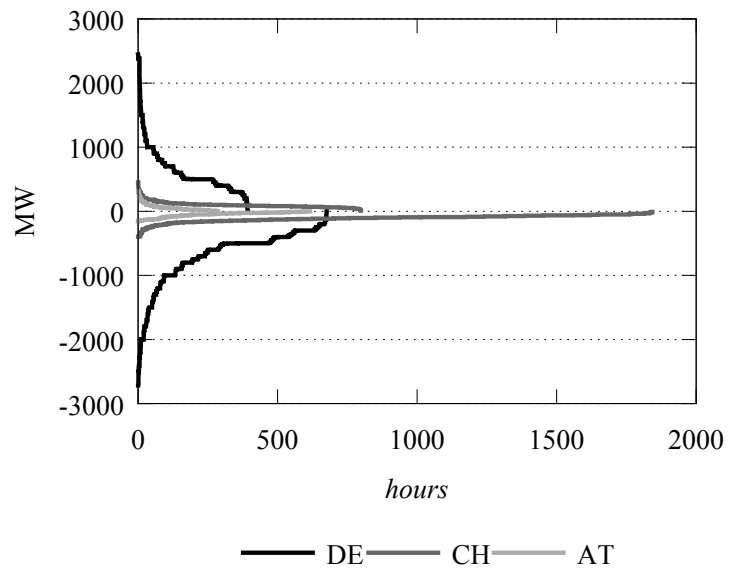

(b) Tertiary Capacity

Figure 3: Balancing energy call duration curves of 2013.

Bundesamt für Energie BFE (2013). Load time series for all regions are taken from ENTSO-E (2013a).

The power plant data for Germany is based on the DIW Data Documentation 72 by Egerer et al. (2014), and for Austria and Switzerland based on Platts (2011) as well as additional data from BFE (2014), BNetzA (2014), and Verbund (2014). The transfer capacities between regions are based on NTC values from ENTSO-E (2010). Cost assumptions for fuels and the $\mathrm{CO}_{2}$ price are based on Egerer et al. (2014). Power plant characteristics are derived from the DIW Data Documentation 68 by Schroeder et al. (2013).

Data for necessary reserved balancing power and called balancing energy is taken from the official platform of the four German TSOs Regelleistung.net (2013) for Germany and from Swissgrid (2013) for Switzerland and E-Control (2013) for Austria. Figure 3 shows the duration curves of balancing energy calls from 2013. Values above zero represent positive calls, whereas negative values represent negative balancing energy calls. The figures show that the balancing energy demand for SC can reach above $2000 \mathrm{MW}$ and below $-2000 \mathrm{MW}$ in Germany. The balancing energy need in Austria and Switzerland is smaller, here the SC balancing energy calls do not exceed $\pm 400 \mathrm{MW}$ for Switzerland and $\pm 200 \mathrm{MW}$ for Austria. While calls for secondary balancing energy occur throughout the year, tertiary capacity is used less frequently. At the same time the countries' peak calls for tertiary balancing energy are higher. Comparing these numbers to the peak load of about $84 \mathrm{GW}$ and an overall energy demand of about 535 TWh in Germany shows that the energy called on the balancing energy markets is - by its nature - relatively small. The same holds true for Austria with a peak demand of about 10.2 GW and a yearly consumption of $66 \mathrm{TWh}$ as well as for Switzerland with a peak demand of $9.8 \mathrm{GW}$ and a yearly consumption of $62 \mathrm{TWh}$.

In the calculations the balancing time series is aggregated from quarter hours to full hours, as the model's time resolution is one hour. This is achieved by taking the maximum 
call of each hour and ensures that the necessary ramps that occur when balancing energy is called are also realized in our model. However this slightly overestimates the total amount of called balancing energy. The call time series for the entire year is also used to generate blocks with specific call frequencies for each country, product, and direction. In this application we use ten different blocks for each balancing product. These call frequencies are used to estimate the call cost when reserving balancing power. See section 3 for an explanation of call frequencies and blocks.

\section{Results and Discussion}

\subsection{Overall Results}

Our results indicate that increased cooperation in the provision of balancing power and energy leads to a reduction in total cost, as expected. However these reductions are small in comparison to the total market volume of the electricity market as depicted in table 2 . The most beneficial scenario Full Cooperation leads to savings of up to $€ 36.8$ million per year. Imbalance Netting and Joint Activation lead to $€ 11.4$ million savings per year.

These relatively small savings in comparison to the total cost are mainly caused by the small volumes of reserved balancing capacity in comparison to the spot market load. Furthermore only a small fraction of these capacities is called and causes direct generation costs.

There are almost no differences in system cost between the scenarios Imbalance Netting and Joint Activation. This is caused by the reservation procedure, which is done regionally within each country. In the scenario Joint Activation, no cross-border capacity reservation for balancing energy takes place. When calls of balancing energy occur, the respective reservation constraint is deactivated in order to allow for cross-border activation. Furthermore imbalance netting is also conducted in both scenarios. Therefore cost savings between these scenarios can only be achieved during the call of balancing energy where little additional potential can be raised. However, these costs are comparatively small and the change caused by this factor is not visible when comparing total system costs. While the cost savings of these two scenarios are almost identical, the setup costs will vary significantly as Joint Activation requires higher coordination between the participating TSOs. Therefore the coordinating measures Imbalance Netting or Full Cooperation should be favored compared to Joint Activation.

Table 2: Total Cost and Savings in the different scenarios

\begin{tabular}{rrr}
\hline million $€$ per year & Total Cost & Savings \\
\hline No Cooperation & $10,119.83$ & \\
Imbalance Netting & $10,108.42$ & 11.41 \\
Joint Activation & $10,108.38$ & 11.46 \\
Full Cooperation & $10,083.04$ & 36.80 \\
\hline
\end{tabular}




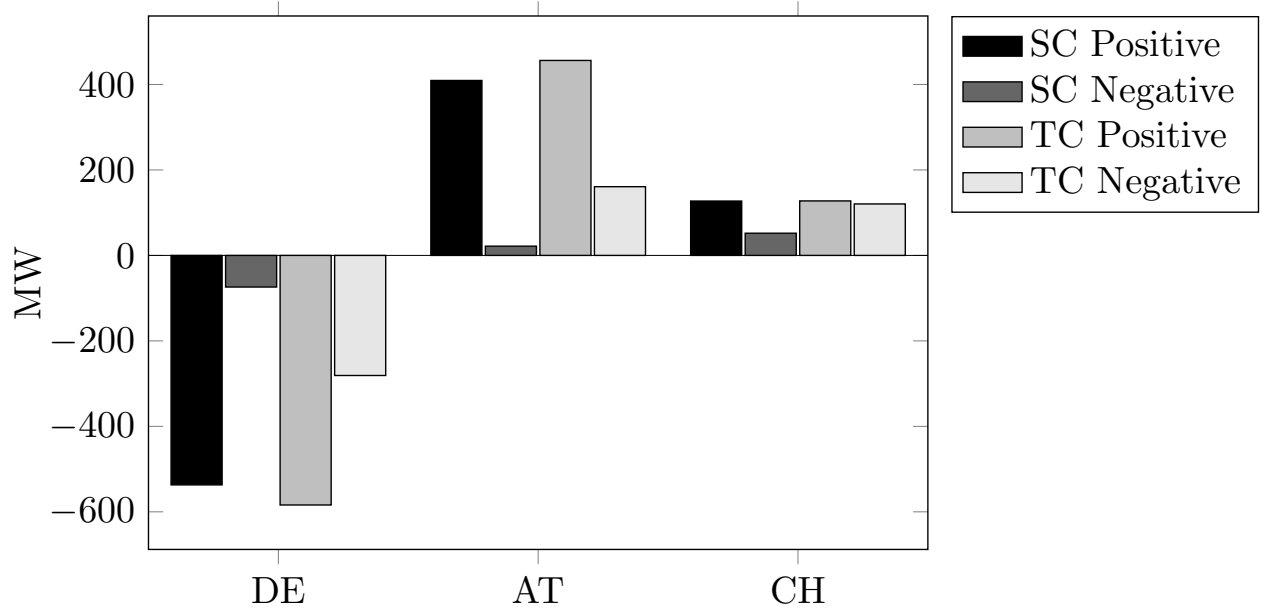

Figure 4: Change of reserved capacity in Full Cooperation compared to No Cooperation

\subsection{Relative Effects on Countries}

When regional cooperation is in place a great impact on the amounts of reserved and called balancing capacities can be observed. The scenario Full Cooperation - the only one that allows for inter-regional reservation - shows drastic changes of reserved capacities within the regions (figure (4). The model results suggest, that balancing capacity is shifted from Germany to Austria and Switzerland. This is especially visible for positive secondary and tertiary reserves where more than $20 \%$ of the capacity is shifted out of Germany. For negative reservation the decrease in Germany is less than 10\%. Due to the overall smaller balancing reserves requirement in Austria, this results in an increase of reserved capacity in Austria of $196 \%$ for positive secondary and $139 \%$ for tertiary reserves. Reasons for these results are the differences in the generation portfolios between Germany and Austria/Switzerland. Germany's generation portfolio contains more fossil fueled generation capacities to serve its base load than its neighbors. Austria and Switzerland mainly rely on hydro power with the assumption of zero marginal cost. Withheld generation capacity from run-of-river power plants is lost in our model as it can not be stored. Therefore it is not beneficial to reserve positive capacity with this technology. However it is beneficial to reserve negative capacity as these plants have no assumed minimum generation level. Due to their marginal cost close to zero, run-of-river power plants are nearly always in the market. It is the other way around with (pumped) storage plants, where unused water is not lost. Hence it is especially beneficial to use storage for positive reserve capacities. Furthermore these plants also do not have minimum generation constraints and can can be started very quickly in our model.

The relative change in called energy shown in figure 5 shows a similar picture. This figure depicts the average change of called balancing energy per country and product. As balancing energy calls are not constant but occur dispersed over time the values shown are about one order of magnitude smaller than in figure 4. The shift in Germany's called 


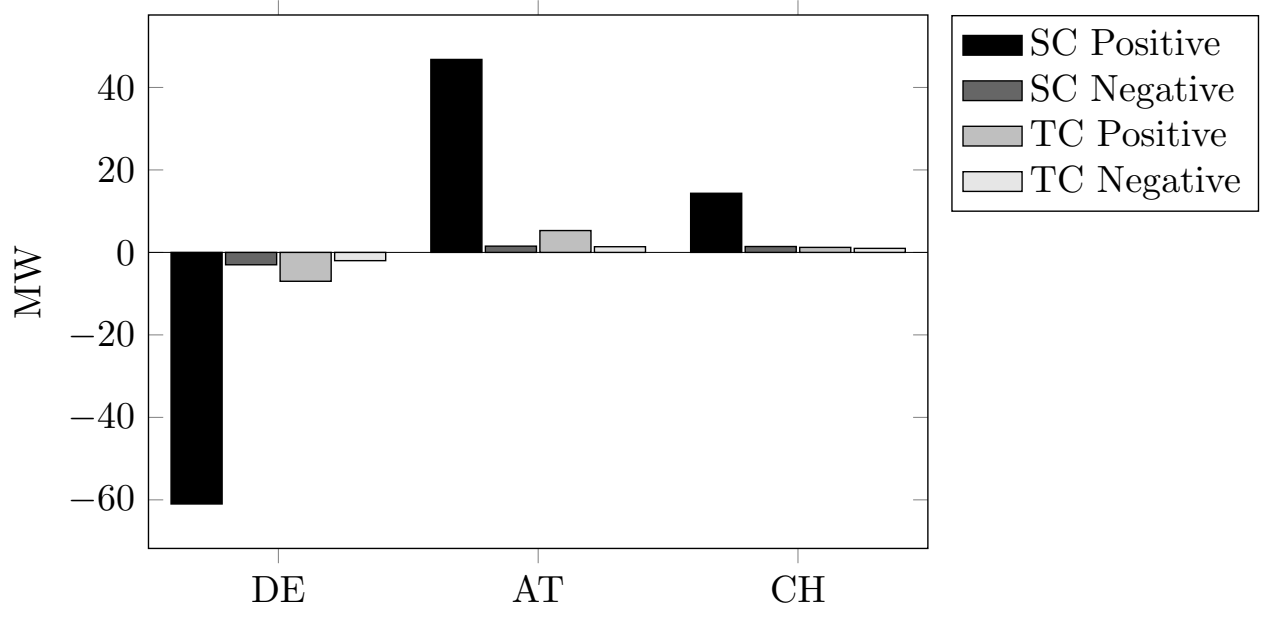

Figure 5: Change of average called energy in Full Cooperation compared to Imbalance Netting

positive secondary and tertiary control account for an decrease of $25 \%$. This leads to an increase of $118 \%$ of the called positive secondary reserves for Austria. Please note that here the Full Cooperation scenario is compared to the Joint Activation scenario, as due to the imbalance netting the baseline for balancing energy calls would be different when comparing to the No Cooperation scenario.

These results are predetermined by the reservation shown in figure 4, as balancing energy can only be called from earlier reserved units. Therefore the called energy from Austria and Switzerland rises in comparison to the Joint Activation scenario. For positive $\mathrm{SC}$, Switzerland shows a higher increase of called reserves than the prior increase in reserved energy while for positive TC Austria shows a greater increase in comparison to the prior reservation. The decrease in called balancing energy in Germany is in line with the decrease in reserved balancing capacity.

It is not only the amount of reserved capacity that allows for insights into a theoretically cost-optimal allocation of reserve capacity, but also the technologies that are used for capacity reservation. Figure 6 shows the reserved positive and negative SC in Austria for the No Cooperation scenario and the Full Cooperation scenario over the four weeks modeled. The values shown are aggregated by fuel type. For positive SC, primarily pumped storage is used, while for negative SC mainly run-of-river plants are used. This is in line with the spot market results where run-of-river plants are always dispatched in the spot market, which is a prerequisite to deliver negative reserves. For positive reservation the model uses primarily (pumped) storage plants. It is beneficial for the system to use storage plants for positive capacity reservation, as the actual use of this capacity is not known during the reservation phase. However, there are single hours where positive reserve is provided by power plants with very high marginal cost, as during these times it is more efficient to reserve these plants and to use the storage plants in the spot market.

Compared to the No Cooperation scenario, the reservation of positive balancing capacity 


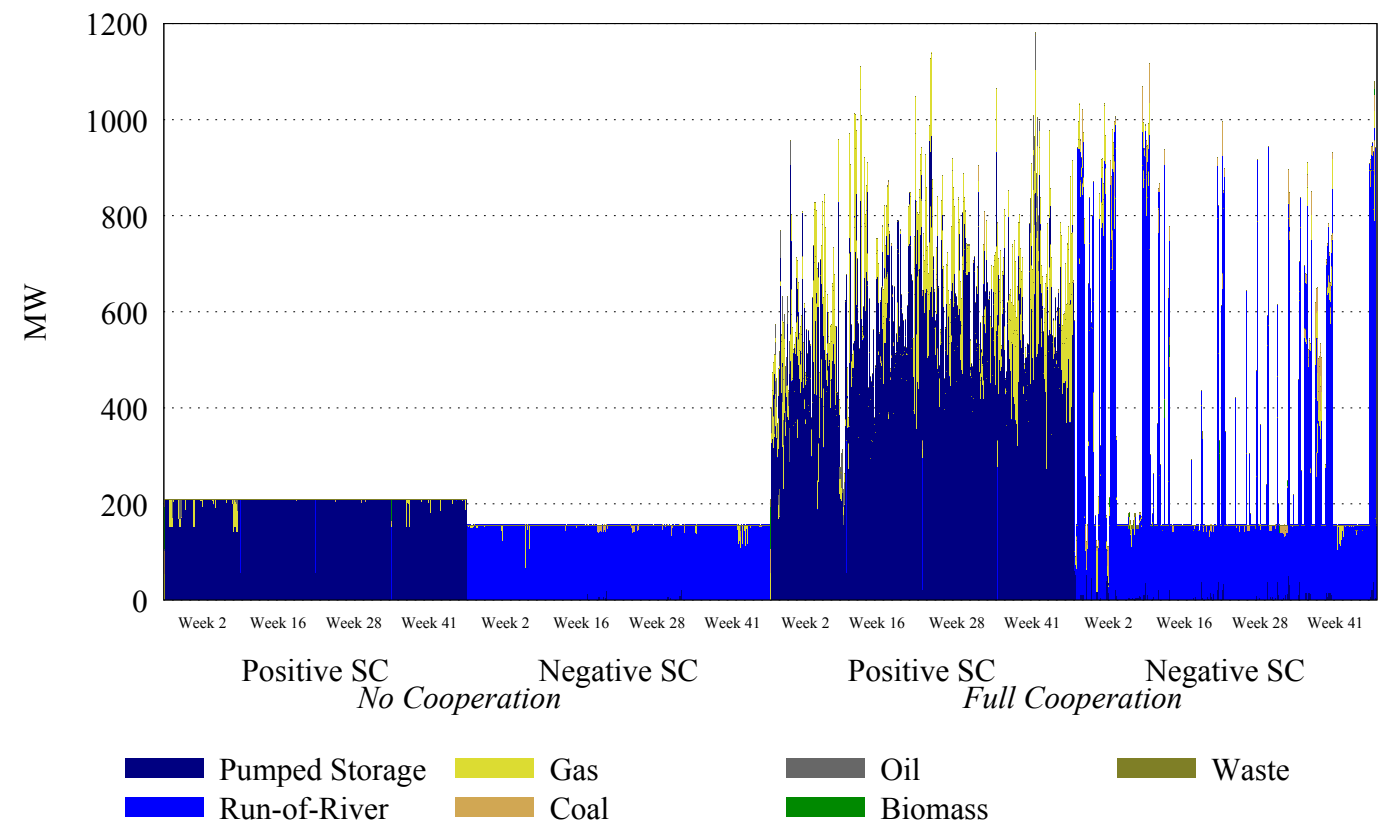

Figure 6: Reserved capacity for secondary control reserve in Austria in the No Cooperation and in the Full Cooperation scenarios

increases significantly in the Full Cooperation scenario. Here additional pumped hydro storage capacity is used, while the reservation of other technologies, especially combined cycle gas turbines increases as well. This shows that the model makes use of allowed reservation across borders. The total amount of reserved capacity for the entire region remains constant. For negative capacity, the amount of capacity reservation in Austria is slightly increased. The reservation baseline from the No Cooperation scenario remains, but several large spikes can be observed, where capacity reservation is shifted towards Austria. As this model allows for hourly capacity reservation, these spikes might not occur with longer reservation periods. For negative capacity mainly run-of-river power plants are used.

As shown above, efficiency gains can be obtained when shifting some positive and negative capacity reservation to Austria, which would otherwise be reserved in Germany if no cooperation were possible. A similar pattern can be observed when comparing these two scenarios for Switzerland. These shifts of reserved balancing capacity and called balancing energy might incur distributional effects and shifts between producers' and consumers' rents. Rents could also be transferred between producers in different countries and price effects could also occur on the spot market. For example, German customers could profit from lower reserve and spot prices, while prices in Austria might rise. Austrian producers could at the same time increase their rents. Therefore a compensation mechanism might be necessary to incentivize all participants to join such a cooperation. 


\subsection{Model Limitations}

Our model allows capacity reservation on an hourly basis. Together with the neglection of uncertainty of renewable energies infeed and load realization, a perfect adjustment of the reserved capacities is possible. Thus, the true cost of the balancing energy system are likely underestimated. Furthermore the benefits shown by our results are generated under the assumption of a social planner. We abstract from any strategic behavior that the market participants might want to apply, which would lead to higher prices on the spot and balancing markets and would increase costs. Within our model application no core share of balancing capacity per region was modeled and security of supply was not taken into account. Thus the costs described in this section are indicative and not comparable to actual market results.

\section{Conclusion}

In this paper we analyze model based regional cooperation scenarios on the balancing energy market. The basis of our analysis stems from the "Network Code Electricity Balancing" by the association of Europe's transmission system operators ENTSO-E. It introduces various regularities to increase cross-border exchange of balancing services and should lead to lower overall balancing cost.

We estimate the efficiency increases for different levels of regional cooperation on the balancing energy markets of Switzerland, Austria and Germany. The model results show that cost decreases can be obtained with increasing levels of coordination. Little differences between Imbalance Netting and Joint Activation exist, while the set-up costs of Joint Activation will be higher than Imbalance Netting. Thus, Imbalance Netting or Full Cooperation seem to be the favorable forms of cooperation. These cost savings are relatively small compared to the total system cost, as the overall size of the spot market is much larger than the balancing services market. At the same time relatively large shifts of capacity reservation towards Austria and Switzerland occur when coordinated procurement and cross-border capacity reservation are in place. These shifts are largely driven by the countries' different power plant portfolios. This change of capacity reservation could lead to shifts of producers' rents, as well as small influences on the spot markets. Therefore the distributional effects on a country's welfare need to be analyzed carefully when planning to join such a cooperation, as welfare effects might not be visible on the balancing but for example on the spot market. Furthermore a compensation mechanism might be needed in order to incentivize possibly disadvantaged parties to join a cooperation.

\section{Acknowledgments}

The authors would like to thank Alexander Weber, Friedrich Kunz, and Christian von Hirschhausen for valuable comments and support. 


\section{References}

50Hertz. 2013. Netzkennzahlen.

Abbasy, A., Van der Veen, R. A C, \& Hakvoort, R.A. 2009 (June). Effect of integrating regulating power markets of Northern Europe on total balancing costs. Pages 1-7 of: PowerTech, 2009 IEEE Bucharest.

Abbasy, A., van der Veen, R., \& Hakvoort, R. 2011 (May). Possible effects of balancing market integration on performance of the individual markets. Pages 608-613 of: Energy Market (EEM), 2011 8th International Conference on the European.

ACER. 2012. Framework Guidelines on Electricity Balancing. Tech. rept. Agency foro Cooperation of Energy Regulators.

ACER. 2014. Opinion of the Agency for the cooperation of energy regulators No 07/2014.

Amprion. 2013. Netzkennzahlen.

BFE. 2014. Statistik der Wasserkraftanlagen der Schweiz.

BNetzA. 2014. Kraftwerksliste der Bundesnetzagentur, Stand 02.04.2014.

Borggrefe, Frieder, \& Neuhoff, Karsten. 2011. Balancing and intraday market design: Options for wind integration. Tech. rept. Discussion Papers, German Institute for Economic Research, DIW Berlin.

Bundesamt für Energie BFE. 2013. Energiestatistiken.

dena. 2014. dena-Studie Systemdienstleistungen 2013. dena-studie Endbericht. dena.

DG ENER, Directorate-General-Energy. 2013. Impact Assessment on European Electricity Balancing Market.

E-Control. 2013. Betriebsstatistik 2013.

Egerer, Jonas, Kunz, Friedrich, Gerbaulet, Clemens, Weber, Alexander, Weibezahn, Jens, Ihlenburg, Richard, Reinhard, Benjamin, \& von Hirschhausen, Christian. 2014. Data Documentation - Modeling the German (2012) and European (2011) Electricity Sector. DIW Data Documentation.

ENTSO-E. 2010. NTC Matrix.

ENTSO-E. 2013a. ENTSO-E Consumption Data.

ENTSO-E. 2013b. Network Code on Electricity Balancing.

Farahmand, H., \& Doorman, G.L. 2012. Balancing market integration in the Northern European continent. Applied Energy, 96(0), 316 - 326. Smart Grids. 
Farahmand, H., Aigner, T., Doorman, G.L., Korpas, M., \& Huertas-Hernando, D. 2012. Balancing Market Integration in the Northern European Continent: A 2030 Case Study. Sustainable Energy, IEEE Transactions on, 3(4), 918-930.

Gebrekiros, Y.T., Doorman, G.L., Farahmand, H., \& Jaehnert, S. 2013 (June). Benefits of cross-border reserve procurement based on pre-allocation of transmission capacity. Pages 1-6 of: PowerTech (POWERTECH), 2013 IEEE Grenoble.

Gerbaulet, Clemens, Hankel, Lisa, Laurisch, Lilian Charlotte, Lorenz, Casimir, Sander, Aram, Schröder, Andreas, \& Zerrahn, Alexander. 2012. Benefits of Regional Cooperation in Providing Balancing Power. Electricity Markets Working Papers.

Hirth, Lion, \& Ziegenhagen, Inka. 2013. Control Power and Variable Renewables: A Glimpse at German Data. Nota di Lavoro 46.2013. Fondazione Eni Enrico Mattei.

Holttinen, Hannele, Meibom, Peter, Orths, Antje, Lange, Bernhard, OMalley, Mark, Tande, John Olav, Estanqueiro, Ana, Gomez, Emilio, Söder, Lennart, Strbac, Goran, Smith, J Charles, \& van Hulle, Frans. 2011. Impacts of large amounts of wind power on design and operation of power systems. Wind Energy, 14(2), 179 - 192.

IGCC, International Grid Control Cooperation. 2014. Information on grid control cooperation and international development.

Jaehnert, S., \& Doorman, G.L. 2010. Modelling an integrated northern European regulating power market based on a common day-ahead market. In: conference paper, IAAE's Rio 2010 International Conference, Rio de Janeiro, Brazil.

Platts. 2011. World Electric Power Plants Database.

Regelleistung.net. 2013. Data for control reserve.

Schroeder, An., Kunz, F., Meiss, J., Mendelevitch, R., \& von Hirschhausen, C. 2013. Current and Prospective Costs of Electricity Generation until 2050. DIW Data Documentation.

Swissgrid. 2013. Swissgrid - Regelenergie.

TenneT. 2013. Netzkennzahlen.

Tractebel. 2009. Study of the Interactions and Dependencies of Balancing Markets, Intraday Trade and Automatically Activated Reserves. Tech. rept. TREN/C2/84/2007. Tractebel.

TransnetBW. 2013. Kennzahlen.

Van der Veen, R. A C, Abbasy, A., \& Hakvoort, R.A. 2010 (June). A qualitative analysis of main cross-border balancing arrangements. Pages 1-6 of: Energy Market (EEM), 2010 \%th International Conference on the European. 
Van der Veen, R. A C, Abbasy, A., \& Hakvoort, R.A. 2011 (May). Analysis of the impact of cross-border balancing arrangements for Northern Europe. Pages 653-658 of: Energy Market (EEM), 2011 8th International Conference on the European.

Vandezande, L., Meeus, L., Saguan, M., Glachant, J. M, \& Belmans, R. 2008. Implementation of cross-border balancing in Europe. Pages 1-6 of: 2008 First International Conference on Infrastructure Systems and Services: Building Networks for a Brighter Future (INFRA). IEEE.

Verbund. 2014. Unsere Kraftwerke.

Wieschhaus, Lars, \& Weigt, Hannes. 2008 (Oct.). Economic Interactions between Electricity Reserve Markets and Wholesale Electricity Markets. Tech. rept. Dresden University of Technology. 


\section{Appendix}

\section{Nomenclature}

\section{Sets}

$t, t t \quad$ Time

$r \quad$ Region

$p \quad$ Power plants

$c \quad$ Subset of conventional power plants

$u \quad$ Subset of fast starting power plants

$o \quad$ Subset of must-run power plants

$s \quad$ Subset of PSP powerplants

bl Blocks of balancing power

$b \quad$ Balancing power product

\section{Parameters}

\begin{tabular}{|c|c|}
\hline$c_{p}^{\text {start }}$ & Cost per start-up \\
\hline$c_{p}^{\text {down }}$ & Cost per shut-down \\
\hline$m c_{c}$ & Marginal generation costs \\
\hline$g_{p}^{\max }$ & Maximum generation \\
\hline$g_{p}^{\min }$ & Minimum generation if online \\
\hline$g_{t}^{\text {sol }}$ & Solar energy feed-in \\
\hline$g_{t}^{\text {wind }}$ & Wind energy feed-in \\
\hline$g_{t}^{b i o}$ & Biomass energy feed-in \\
\hline$r_{p}^{\text {down }}$ & Maximum ramping down speed [\% per hour] \\
\hline$r_{p}^{u p}$ & Maximum ramping up speed [\% per hour] \\
\hline$q_{t}^{\text {spot }}$ & Electricity load \\
\hline$q_{b, b l, r, t}^{r e s v}$ & Total amount of negative balancing power needed \\
\hline$q_{b, b l, r, t}^{\text {resu }}$ & Total amount of positive balancing power \\
\hline$q_{b, r, t}^{\text {call, neg }}$ & Total Negative call in per region, time, and product \\
\hline$q_{b, r, t}^{\text {call,pos }}$ & Total Positive call in per region, time, and product \\
\hline$f_{r, r r}^{\max }$ & Max flow \\
\hline$f r q_{b l, b}$ & Call frequency of balancing reserve in a specific block \\
\hline$l_{s}^{\max }$ & Maximum storage level \\
\hline$l_{s}^{\min }$ & Minimum storage level \\
\hline$v_{s}^{\max }$ & Maximum storage release \\
\hline$w_{s}^{\max }$ & Maximum storage loading \\
\hline & Storage efficiency \\
\hline$g_{s, t}^{n a t}$ & Natural inflow into storage \\
\hline
\end{tabular}




\section{Variables}

Cost

Cost $^{\text {gen }}$

Objective value: total cost

Costresv

Generation cost

Cost ${ }^{\text {call }}$

Total balancing reservation cost

Cost start $^{\text {st }}$

Total balancing call cost

Cost down

Total start up cost

$G_{c, t}^{\text {spot }}$

$G_{\text {resv,pos }}^{\text {rest }}$

$G_{p, t, b l, b}$

$G_{p, t, b l, b}^{r e s u, h e g}$

$G^{r e s v, p o s, A}$

$G_{s, t, b l, b}^{r e s v, p o s, A}$

$G_{s, t b l, b}^{r e s v, P}$

$G^{\text {resv, neg, } A}$

$G_{s, t, b l, b}^{r e s v i n}$

$G_{s, t, b l, b}^{r, e s v, \text { neg }, P}$

$G_{b, p a l l, p o s}$

$G_{b, p, t}^{c a l l}, p$

$G_{b, p, t}^{c a l l, \text { neg }}$

$G_{b, p, t}$

$F_{r, r r, t}^{s p, t}$

$F_{b, b l, r, r, t, t}^{r e s v, p o s}$

$F_{b, b l, n e g}^{r e s u}$

$F_{b a l l, p o s}^{\text {cal }}$

$F_{b, r, r r, t}^{c a l l, p o s}$

$F_{b, r, r, t}^{c a l l, \text { neg }}$

$F_{b e s v, p o s, g e 0}^{r e n}$

$F_{b, b l, r, r r, t}^{r, t}$

$F_{b e s, \text { pos }, l e 0}$

$F_{b, b l, r, r r, t}$

$F_{b, b l, r, r r, t}^{r \text { resveg }, \text {, } 0}$

$F_{b, b s, \text { neg, le } 0}^{\text {res }}$

$F_{b, b l, r, r r, t}^{r e s, n e g}$

Frq $q_{u, t}^{\max }$

$P S P_{s, t}^{\text {discard }}$

Total shut down cost

Conventional generation in MW

Positive reserved balancing power assigned to a plant

Negative reserved balancing power assigned to a plant

Positive reserved balancing power of a PSP (active $=$ more generation)

Positive reserved balancing power of a PSP (passive = less pumping)

Negative reserved balancing power of a PSP (active $=$ more pumping)

Negative reserved balancing power of a PSP (passive $=$ less generation)

Positive called balancing energy

Negative called balancing energy

Spot market flow

Reservation of positive balancing flow

Reservation of negative balancing flow

Positive balancing flow

Negative balancing flow

Positive part of the reservation of positive balancing flow

Negative part of the reservation of positive balancing flow

Positive part of the reservation of negative balancing flow

Negative part of the reservation of negative balancing flow

Highest possible Call Frequency in specific hour

Discard of excess water

$P S P_{s, t}^{D}$

$P S P_{s, t}^{G}$

$P S P_{s, t}^{L}$

Storage loading (pumping)

Storage release (generation)

Storage level

\section{Binary Variables}

$O N_{c, t} \quad$ Plant status

$U P_{c, t} \quad$ Plant startup variable

$D N_{c, t} \quad$ Plant shutdown variable

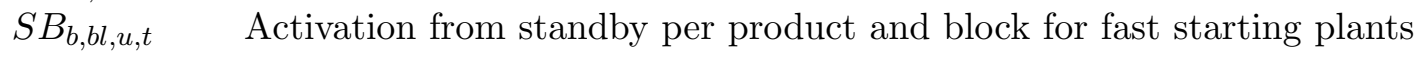

